\title{
THE $R_{\infty}$ PROPERTY FOR INFRA-NILMANIFOLDS
}

\author{
Karel Dekimpe - Bram De Rock - Pieter Penninckx
}

\begin{abstract}
In this paper, we investigate the finiteness of the Reidemeister number $R(f)$ of a selfmap $f: M \rightarrow M$ on an infra-nilmanifold $M$. We show that the Reidemeister number of an Anosov diffeomorphism on an infra-nilmanifold is always finite. A manifold $M$ is said to have the $R_{\infty}$ property if $R(f)=\infty$ for every homeomorphism $f: M \rightarrow M$. We show that every non-orientable generalised Hantzsche-Wendt manifold has the $R_{\infty}$ property. For an orientable Hantzsche-Wendt manifold $M$, we formulate a criterion, in terms of an associated graph, for $M$ to have the $R_{\infty}$ property.
\end{abstract}

\section{Introduction}

To a continuous map $f: M \rightarrow M$ on a closed manifold $M$, three numbers are assigned that are of particular interest in fixed point theory: the Lefschetz number $L(f)$, the Nielsen number $N(f)$ and the Reidemeister number $R(f)$. The Nielsen number $N(f)$ holds the most information on the fixed points of $f$, but is the hardest to calculate. In order to express the Nielsen number in terms of the Lefschetz number and the Reidemeister number, relations between these numbers have been extensively studied.

2000 Mathematics Subject Classification. Primary 55M20; Secondary 20F34.

Key words and phrases. Reidemeister number, infra-nilmanifolds, Hantzsche-Wendt manifolds.

This research was supported by: the Research Grant of the Research Foundation-Flanders (FWO): 1.5.026.05, the Research Programme of the Research Foundation-Flanders (FWO): G.0570.06 and the Research Fund of the Katholieke Universiteit Leuven.

The third named author is supported by Ph. D. fellowship of the Research Foundation Flanders (FWO). 
A closed manifold $M$ is said to be a Jiang-type space if

$$
N(f)=\left\{\begin{array}{ll}
0 & \text { if } L(f)=0, \\
R(f) & \text { if } L(f) \neq 0,
\end{array} \quad \text { for all continuous maps } f: M \rightarrow M\right.
$$

Note that nilmanifolds are Jiang-type spaces. Indeed, for any selfmap $f: M \rightarrow M$ on a nilmanifold, $N(f)=|L(f)|$ by Anosov [1] and $L(f) \neq 0$ implies $N(f)=$ $R(f)$ by [17].

In the light of the relation (1.1), the finiteness of the Reidemeister number is important. Indeed, if the Reidemeister number is infinite, the Nielsen number, which is always finite, cannot equal the Reidemeister number. As a complement to the relation (1.1), Wong defines the $R_{\infty}$ property:

Definition 1.1. A manifold $M$ has the $R_{\infty}$ property if $R(f)=\infty$ for every homeomorphism $f: M \rightarrow M$.

Since the Reidemeister number can be defined at the level of the fundamental group, the $R_{\infty}$ property can be studied at the level of the fundamental group as well. This has been done for a large and growing number of classes of manifolds (see for instance [20] and the references therein).

In this article, we search for classes of infra-nilmanifolds that have the $R_{\infty}$ property. As a first step, we give a criterion for the finiteness of the Reidemeister number of a given selfmap on an infra-nilmanifold. We obtain that an infranilmanifold that admits an Anosov diffeomorphism cannot have the $R_{\infty}$ property since we show that the Reidemeister number of an Anosov diffeomorphism on an infra-nilmanifold is always finite. For closed flat manifolds, the holonomy representation determines the existence of an Anosov diffeomorphism ([18]). This guides us to a condition on the holonomy representation of a closed flat manifold $M$ under which $M$ has the $R_{\infty}$ property.

The generalised Hantzsche-Wendt manifolds (or GHW manifolds for short) form a well described class of infra-nilmanifolds for which we obtain precise results. We show that a non-orientable GHW manifold always has the $R_{\infty}$ property. The orientable Hantzsche-Wendt manifolds (or HW manifold for short) are studied by Miatello and Rossetti in [16], where a graph is associated to an HW manifold that is in "standard form" (see Section 2.4). Since every HW manifold is homeomorphic to one in standard form, we can formulate, in terms of an associated graph, a criterion for an HW manifold to have the $R_{\infty}$ property. To be precise, we show that an HW manifold $M$ does not have the $R_{\infty}$ property if and only if $M$ is homeomorphic to an HW manifold of which the associated graph is circulant. 


\section{Preliminaries}

In this section, we introduce the basic notions needed for the formulation of the results. For more information on the Lefschetz number, the Nielsen number and the Reidemeister number, we refer to [3], [7], [10], [11]. For more information on infra-nilmanifolds, we refer to [4].

2.1. Lefschetz number, Reidemeister number and Nielsen number. Let $f: M \rightarrow M$ be a continuous map on a closed manifold $M$. The Lefschetz number $L(f)$ is defined by

$$
L(f)=\sum_{i}(-1)^{i} \operatorname{Trace}\left(f_{*}: H_{i}(M, \mathbb{Q}) \rightarrow H_{i}(M, \mathbb{Q})\right) .
$$

This number is of interest in fixed point theory since $L(f) \neq 0$ implies that $f$ has a fixed point. Because the Lefschetz number is invariant under homotopy, $L(f) \neq 0$ implies that any map homotopic to $f$ has a fixed point.

The Reidemeister number can be defined at the level of the fundamental group. The continuous map $f: M \rightarrow M$ induces a morphism $f_{\sharp}: \pi_{1}(M) \rightarrow \pi_{1}(M)$ on the fundamental group $\pi_{1}(M)$ of $M$. We say that two elements $\alpha, \beta \in \pi_{1}(M)$ are $f_{\sharp}$-conjugated if there exists $\gamma \in \pi_{1}(M)$ such that $\beta=\gamma \alpha f_{\sharp}(\gamma)^{-1}$. The $f_{\sharp^{-}}$ conjugacy class $\left\{\gamma \alpha f_{\sharp}(\gamma)^{-1} \mid \gamma \in \pi_{1}(M)\right\}$ of $\alpha$ is called a Reidemeister class of $f$. The number of Reidemeister classes is called the Reidemeister number $R(f)$ of $f$.

Nielsen's approach to estimate the number of fixed points of $f$ is of a more geometric nature. Let $\widetilde{M}$ be the universal covering space of $M$ and let $p: \widetilde{M} \rightarrow M$ be the covering map. We define an equivalence relation on the set of lifts of $f$ : two lifts $\widetilde{f}^{a}$ and $\widetilde{f}^{b}$ are equivalent if and only if there is a covering transformation $\gamma: \widetilde{M} \rightarrow \widetilde{M}$ such that $\widetilde{f}^{b}=\gamma \circ \widetilde{f}^{a} \circ \gamma^{-1}$. The equivalence classes are called lifting classes. The number of lifting classes equals the Reidemeister number. A lifting class $[\widetilde{f}]$ containing a lift $\tilde{f}$ gives rise to a so called fixed point class $p(\operatorname{Fix}(\tilde{f}))$. To every fixed point class, an integer index is assigned. When this index differs from zero, we call the fixed point class essential. The Nielsen number $N(f)$ is by definition the number of essential fixed point classes of $f$. The interest in the Nielsen number arises from the fact that $N(f)$ is a lower bound of the number of fixed points of $f$. Because the Nielsen number is invariant under homotopy, every map homotopic to $f$ has at least $N(f)$ fixed points.

2.2. Infra-nilmanifolds. Let $G$ be a connected, simply connected, nilpotent Lie group of dimension $n$. We denote by $\operatorname{Endo}(G)$ the semigroup of all endomorphisms of $G$. The semigroup $\operatorname{Endo}(G)$ acts naturally on $G$ and contains $\operatorname{Aut}(G)$ as a subgroup. We use $\operatorname{aff}(G)$ to denote the semigroup $G \rtimes \operatorname{Endo}(G)$, which is $G \times \operatorname{Endo}(G)$ as a set, with multiplication defined by $\left(d_{1}, D_{1}\right)\left(d_{2}, D_{2}\right)=$ $\left(d_{1} D_{1}\left(d_{2}\right), D_{1} D_{2}\right)$. An element $(d, D)$ of aff $(G)$ is called an affine endomorphism of $G$ and it maps $g \in G$ to $d D(g)$. We can think of multiplication in $\operatorname{aff}(G)$ as 
composition of maps. When $D$ is an automorphism of $G$ (that is $D \in \operatorname{Aut}(G)$ ), an affine endomorphism $(d, D)$ is invertible as a selfmap on $G$ and as an element of the semigroup $\operatorname{aff}(G)$. We use $\operatorname{Aff}(G)$ to denote the invertible affine endomorphisms of $G$, it equals $G \rtimes \operatorname{Aut}(G)$ and is a subgroup of $\operatorname{aff}(G)$.

An almost-crystallographic group $\Gamma$ is a subgroup of $\operatorname{Aff}(G)$ such that its subgroup of pure translations $\Gamma \cap G$ is a uniform lattice of $G$ and $\Gamma \cap G$ is of finite index in $\Gamma$. We obtain an action of an almost-crystallographic group $\Gamma$ on $G$ by restricting the action of $\operatorname{Aff}(G)$ on $G$ to an action of $\Gamma$ on $G$. Then the orbit space $\Gamma \backslash G$ is compact. When $\Gamma$ is torsion free, we call $\Gamma$ an almost-Bieberbach group and then the orbit space $\Gamma \backslash G$ is a closed differentiable manifold; we call it an infra-nilmanifold. The fundamental group $\pi_{1}(\Gamma \backslash G)$ of an infra-nilmanifold $\Gamma \backslash G$ is isomorphic to the corresponding almost-Bieberbach group $\Gamma$; in fact $G$ is the universal covering space of $\Gamma \backslash G$ and the group of covering transformations is exactly $\Gamma$. When an almost-Bieberbach group $\Gamma$ lies in $G$, we call $\Gamma \backslash G$ a nilmanifold.

When $G=\mathbb{R}^{n}$, we refer to almost-crystallographic and almost-Bieberbach groups as ( $n$-dimensional) crystallographic and Bieberbach groups, respectively. When $\Gamma$ is a Bieberbach group, $\Gamma \backslash \mathbb{R}^{n}$ is a closed flat manifold. All closed flat manifolds can be obtained in this way.

For an almost-crystallographic group $\Gamma$, the finite quotient $\Gamma /(\Gamma \cap G)$ is isomorphic to the group

$$
F=\{A \in \operatorname{Aut}(G) \mid \exists a \in G:(a, A) \in \Gamma\},
$$

which we call the holonomy group of $\Gamma$. By taking differentials, we obtain a morphism $\rho: F \rightarrow \operatorname{Aut}(\mathfrak{g})$, where $\mathfrak{g}$ is the Lie algebra associated to $G$. By fixing a basis for $\mathfrak{g}$, we obtain a faithful representation

$$
\rho: F \rightarrow \mathrm{GL}_{n}(\mathbb{R}),
$$

which we call the holonomy representation of $\Gamma$. Because of the choice of a basis, this representation is determined up to similarity.

If $\Gamma$ is a crystallographic group, $F$ is a subgroup of $\operatorname{Aut}\left(\Gamma \cap \mathbb{R}^{n}\right)$ and $\Gamma \cap \mathbb{R}^{n}$ is isomorphic to $\mathbb{Z}^{n}$. Then we will always choose a $\mathbb{Z}$-module basis of $\Gamma \cap \mathbb{R}^{n}$ as the basis of $\mathfrak{g}=\mathbb{R}^{n}$ and then $\rho: F \rightarrow \mathrm{GL}_{n}(\mathbb{Z})$. If $\Gamma \cap \mathbb{R}^{n}=\mathbb{Z}^{n}$, we will always choose the standard $\mathbb{Z}$-module basis of $\mathbb{Z}^{n}$ as the basis of $\mathfrak{g}=\mathbb{R}^{n}$. Using this basis, we may then identify a linear map $L: \mathbb{R}^{n} \rightarrow \mathbb{R}^{n}$ with the corresponding matrix.

By the holonomy group and holonomy representation of an infra-nilmanifold, we mean the holonomy group and holonomy representation of the associated almost-Bieberbach group. The holonomy representation holds a lot of information about the infra-nilmanifold. For instance, the holonomy representation of an infra-nilmanifold determines its orientability (see [2, p. 221] and [4, p. 135]): 
Proposition 2.1. Let $M$ be an infra-nilmanifold with holonomy group $F$ and holonomy representation $\rho: F \rightarrow \mathrm{GL}_{n}(\mathbb{R})$. Then $M$ is orientable if and only if $\operatorname{det}(\rho(A))=1$ for every $A \in F$.

2.3. Linearisation of maps on infra-nilmanifolds. Let $\Gamma$ be an almostBieberbach group and $f: M \rightarrow M$ a continuous map on the corresponding infranilmanifold $M=\Gamma \backslash G$. Then $G$ is the universal covering space of $M$. Let $h: G \rightarrow G$ be a continuous map. We say that $h$ induces $f$ if $h$ is a lift of $f$. We say that $h$ is a homotopy lift of $f$ if $h$ is the lift of a map homotopic to $f$. In [14], K. B. Lee proves the following theorem:

THEOREM 2.2. Every continuous map on an infra-nilmanifold has a homotopy lift that is an affine endomorphism.

If $f$ is a homeomorphism, a homotopy lift $(d, D)$ of $f$ is an invertible affine endomorphism: $(d, D) \in \operatorname{Aff}(G)$; moreover, $(d, D)$ belongs to the normaliser $N_{\mathrm{Aff}(G)}(\Gamma)$ of $\Gamma$ in $\operatorname{Aff}(G)$.

2.4. Generalised Hantzsche-Wendt manifolds. An $n$-dimensional closed flat manifold $M$ of which the holonomy group is isomorphic to $\mathbb{Z}_{2}^{n-1}$ is called a generalised Hantzsche-Wendt manifold or GHW manifold for short. For simplicity, we identify the holonomy group with $\mathbb{Z}_{2}^{n-1}$. The corresponding Bieberbach group $\Gamma$ is called a GHW group. When $M$ is orientable, we call $M$ a Hantzsche-Wendt manifold or an HW manifold for short. We call the corresponding Bieberbach group an HW group. It is well known (see [19, p. 1056]) that HW manifolds only exist in odd dimensions.

In [19], Rossetti and Szczepański prove that all GHW groups $\Gamma$ are diagonisable: there exists a $\mathbb{Z}$-module basis of $\Gamma \cap \mathbb{R}^{n}$ such that with respect to that basis, $\rho(x)$ is a diagonal matrix for all $x \in \mathbb{Z}_{2}^{n-1}$, where $\rho: \mathbb{Z}_{2}^{n-1} \rightarrow \mathrm{GL}_{n}(\mathbb{Z})$ is the holonomy representation.

Let us now shortly recall some results from Miatello and Rossetti's article [16]. We say that an HW group is in standard form if it is generated by $\mathbb{Z}^{n}$ and $\left(b_{1}, B_{1}\right), \ldots,\left(b_{n}, B_{n}\right)$, where $B_{i}$ is the diagonal matrix with a 1 on the $i$-th place of the diagonal and -1 on the other places of the diagonal and where $b_{i}=\left(b_{i, 1}, \ldots, b_{i, n}\right) \in\{0,1 / 2\}^{n}$ and $b_{i, i}=1 / 2$ for $i=1, \ldots, n$. Every HW group is isomorphic to an HW group in standard form. To an HW group in standard form, we associate a graph $(V, E)$ with points $V=\{1, \ldots, n\}$ and arrows $E$, where $(i, j) \in E$ (that is: there is an arrow from $i$ to $j$ ) if and only if $b_{j, i} \neq 0$ and $j \neq i$. By a graph $(V, E)$ we mean a simple directed graph consisting of a set of vertices or points $V$ and a set of edges or arrows $E \subset V \times V$ such that $(v, v) \notin E$ for every $v \in V$. We say that two graphs $\left(V_{1}, E_{1}\right)$ and $\left(V_{2}, E_{2}\right)$ are isomorphic or equivalent when there exists a bijection $f: V_{1} \rightarrow V_{2}$ such that for all $v, w \in V_{1}$ 
we have that $(f(v), f(w)) \in E_{2}$ if and only if $(v, w) \in E_{1}$. Miatello and Rossetti prove the following theorems:

TheOREM 2.3. Let $n$ be an odd natural number. A graph $(V, E)$ with vertices $V=\{1, \ldots, n\}$ is the graph associated to an HW group in standard form if and only if both of the following statements hold.

(a) The number of arrows starting from each point is odd.

(b) For every odd order proper subset $\mathcal{V}$ of $V$, there exists $v \in \mathcal{V}$ such that the number of arrows from $v$ to points in $\mathcal{V}$ is even.

THEOREM 2.4. Let $\Gamma_{1}$, respectively $\Gamma_{2}$, be HW groups in standard form with associated graphs $\left(V_{1}, E_{1}\right)$, respectively $\left(V_{2}, E_{2}\right)$. Then $\Gamma_{1}$ and $\Gamma_{2}$ are isomorphic if and only if $\left(V_{1}, E_{1}\right)$ is equivalent to the complement of $\left(V_{2}, E_{2}\right)$ relative to a subset $\mathcal{V}$ of $V_{2}$.

Recall that the complement $\left(V, E^{\prime}\right)$ of a graph $(V, E)$ relative to a set $\mathcal{V} \subset V$ is defined by

$$
E^{\prime}=\{(v, w) \in V \times V \mid v \neq w \text { and }(v, w) \notin E \text { if and only if } v \in \mathcal{V}\} .
$$

\section{The finiteness of the Reidemeister number of a continuous selfmap on an infra-nilmanifold}

In this section, we give a criterion for the finiteness of the Reidemeister number $R(f)$ of a given continuous selfmap $f$ on an infra-nilmanifold. In order to prove this criterion, we need the following two lemmas.

Lemma 3.1. Let $M$ be an infra-nilmanifold with associated almost-Bieberbach group $\Gamma$. Let $f: M \rightarrow M$ be a continuous map. Suppose that $\widetilde{f}=(d, D) \in$ $\operatorname{aff}(G)$ is a lift of $f$. Then there exists a finite index subgroup $\Lambda$ of $\Gamma$ such that $\Lambda \backslash G$ is a nilmanifold $N$ and we have covering maps $\bar{p}: N \rightarrow M$ and $p^{\prime}: G \rightarrow N$ and a continuous map $\bar{f}: N \rightarrow N$ such that the diagram

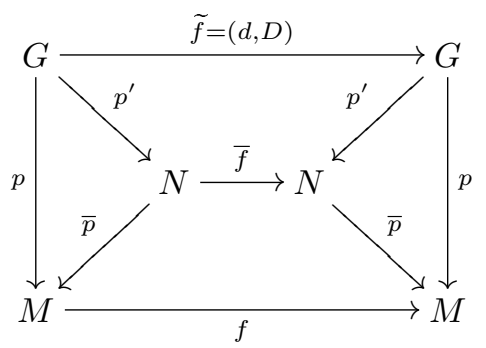

commutes, where $p: G \rightarrow M$ is the natural covering map. Additionally, for any covering transformation $\alpha: G \rightarrow G$ of the covering $p: G \rightarrow M$ (that is, for every 
$\alpha \in \Gamma)$, there exists a covering transformation $\varphi(\alpha): N \rightarrow N$ such that the diagram

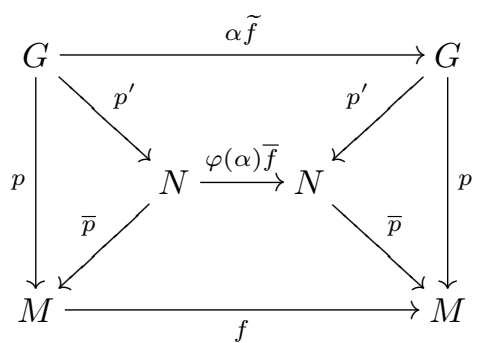

commutes. Moreover, for all $\alpha=(a, A) \in \Gamma$, we have that

$$
L(\varphi(\alpha) \bar{f})=\operatorname{det}\left(\mathbb{1}-(A D)_{*}\right)
$$

where $\mathbb{1}$ denotes the identity matrix and $(A D)_{*}$ is the Lie algebra morphism induced by $A D$.

Proof. Most of this lemma follows from the proof of [13, Lemma 3.2]. For the readers convenience, we recall the main steps.

Let $\Lambda$ be the subgroup of $\Gamma$ generated by $\left\{\alpha^{k} \mid \alpha \in \Gamma\right\}$, where $k$ is the order of the holonomy group. Then by [13, Lemma 3.1], $\Lambda$ is a finite index fully invariant subgroup of $\Gamma$, the quotient space $N=\Lambda \backslash G$ is a nilmanifold and we have natural covering maps $\bar{p}: N \rightarrow M$ and $p^{\prime}: G \rightarrow N$ and a continuous map $\bar{f}: N \rightarrow N$ such that the diagram (3.1) commutes.

The second statement follows by defining $\varphi(\alpha): N \rightarrow N: \Lambda \cdot x \mapsto \Lambda \cdot \alpha(x)$ for any covering transformation $\alpha: G \rightarrow G$ of the covering $p: G \rightarrow M$.

Let $\alpha=(a, A) \in \Gamma$ be a covering transformation. Then $\varphi(\alpha) \bar{f}: N \rightarrow N$ induces a morphism $(\varphi(\alpha) \bar{f})_{\sharp}: \pi_{1}(N) \rightarrow \pi_{1}(N)$ on the fundamental group. Identifying $\pi_{1}(N)$ with $\Lambda$, one can prove that

$$
(\varphi(\alpha) \bar{f})_{\sharp}=\left.(\mu(a A(d)) \circ A D)\right|_{\Lambda},
$$

where by $\mu(a A(d)): G \rightarrow G: x \mapsto a A(d) x(a A(d))^{-1}$ we denote conjugation by $a A(d)$. It follows that $\varphi(\alpha) \bar{f}$ is homotopic to the map $\psi: N \rightarrow N$ induced by $\mu(a A(d)) \circ A D: G \rightarrow G$. By Anosov's article [1],

$$
L(\varphi(\alpha) \bar{f})=\operatorname{det}\left(\mathbb{1}-(\mu(a A(d)) \circ A D)_{*}\right) .
$$

By $\left[13\right.$, Lemma 3.2], $\operatorname{det}\left(\mathbb{1}-(\mu(a A(d)) \circ A D)_{*}\right)=\operatorname{det}\left(\mathbb{1}-(A D)_{*}\right)$. Hence $L(\varphi(\alpha) \bar{f})=\operatorname{det}\left(\mathbb{1}-(A D)_{*}\right)$.

LEMMA 3.2. Let $M$ be an infra-nilmanifold with holonomy representation $\rho: F \rightarrow \mathrm{GL}_{n}(\mathbb{R})$. Let $f: M \rightarrow M$ be a continuous map with homotopy lift $\tilde{f}=$ 
$(d, D) \in \operatorname{aff}(G)$. Then there exists a non-essential fixed point class of $f$ (i.e. $N(f) \neq R(f))$ if and only if there exists $A \in F$ such that $\operatorname{det}\left(\mathbb{1}-(A D)_{*}\right)=0$.

Proof. Without loss of generality, we may assume that $f$ is induced by the homotopy lift $\widetilde{f}=(d, D)$. Let $\Lambda, N, \bar{f}$ and $\varphi$ be as in the previous lemma and let $\Gamma$ be the almost-Bieberbach group associated to $M$.

Suppose $\operatorname{det}\left(\mathbb{1}-(A D)_{*}\right)=0$ for an element $A$ of the holonomy group $F$. Choose $\alpha=(a, A) \in \Gamma$. Then

$$
N(\varphi(\alpha) \bar{f})= \pm L(\varphi(\alpha) \bar{f})= \pm \operatorname{det}\left(\mathbb{1}-(A D)_{*}\right)=0 .
$$

Hence $p^{\prime}(\operatorname{Fix}(\alpha \widetilde{f}))$ is a non-essential fixed point class of $\varphi(\alpha) \bar{f}$. By [12, Remark 2.7], $p(\operatorname{Fix}(\alpha \widetilde{f}))$ is a non-essential fixed point class of $f$.

Conversely suppose $\operatorname{det}\left(\mathbb{1}-(A D)_{*}\right) \neq 0$ for all $A \in F$. Choose a fixed point class $\mathbb{F}$ of $f$. Then $\mathbb{F}=p(\operatorname{Fix}(\alpha \widetilde{f}))$ for an $\alpha=(a, A) \in \Gamma$. Because $N$ is a Jiang-type space and $L(\varphi(\alpha) \bar{f})=\operatorname{det}\left(\mathbb{1}-(A D)_{*}\right) \neq 0$, we have that

$$
N(\varphi(\alpha) \bar{f})=R(\varphi(\alpha) \bar{f})
$$

and every fixed point class of $\varphi(\alpha) \bar{f}$ is essential. Hence also $p^{\prime}(\operatorname{Fix}(\alpha \tilde{f}))$ is an essential fixed point class of $\varphi(\alpha) \bar{f}$. By [12, Remark 2.7], the fixed point class $\mathbb{F}=p(\operatorname{Fix}(\alpha \widetilde{f}))$ is essential.

These two lemmas allow us to prove the following theorem.

THEOREM 3.3. Let $M$ be an infra-nilmanifold with holonomy representation $\rho: F \rightarrow \mathrm{GL}_{n}(\mathbb{R})$. Let $f: M \rightarrow M$ be a continuous map with homotopy lift $\tilde{f}=$ $(d, D)$. Then the following statements are equivalent:

(a) $R(f)=\infty$.

(b) There exists $A \in F$ such that $\operatorname{det}\left(\mathbb{1}-(A D)_{*}\right)=0$.

Proof. We may assume that $f$ is induced by the homotopy lift $\widetilde{f}=(d, D)$. Let $\Lambda, N, \bar{f}$ and $\varphi$ be as in Lemma 3.1 and let $\Gamma$ be the almost-Bieberbach group associated to $M$.

Suppose that $R(f)=\infty$. Because $N(f)<\infty$, we have that $R(f) \neq N(f)$. By the previous lemma, there exists $A \in F$ such that $\operatorname{det}\left(\mathbb{1}-(A D)_{*}\right)=0$.

Conversely suppose there exists $A \in F$ such that $\operatorname{det}\left(\mathbb{1}-(A D)_{*}\right)=0$. Choose $\alpha=(a, A) \in \Gamma$. Then $L(\varphi(\alpha) \bar{f})=\operatorname{det}\left(\mathbb{1}-(A D)_{*}\right)=0$. By [8, Lemma 1], we have that $R(\varphi(\alpha) \bar{f})=\infty$. Now the map

$\psi:\{$ lifting classes of $\varphi(\alpha) \bar{f}\} \rightarrow\{$ lifting classes of $f\}:[\lambda \alpha \widetilde{f}]_{\Lambda} \mapsto[\lambda \alpha \widetilde{f}]_{\Gamma}$

is well defined, where $[\lambda \alpha \widetilde{f}]_{\Lambda}$ is the lifting class of $\varphi(\alpha) \bar{f}$ that contains $\lambda \alpha \widetilde{f}$ (where $\lambda \in \Lambda$ ) and where $[\lambda \alpha \widetilde{f}]_{\Gamma}$ is the lifting class of $f$ containing $\lambda \alpha \widetilde{f}$. To show that $R(f)=\infty$, it suffices to prove that any lifting class $[\lambda \alpha \widetilde{f}]_{\Gamma}$ of $f$ has at 
most $[\Gamma: \Lambda]$ preimages under $\psi$. Choose $\lambda \in \Lambda$ and suppose for a contradiction that

$$
\psi\left(\left[\lambda_{1} \alpha \widetilde{f}\right]_{\Lambda}\right)=\ldots=\psi\left(\left[\lambda_{[\Gamma: \Lambda]} \alpha \widetilde{f}\right]_{\Lambda}\right)=\psi\left(\left[\lambda_{[\Gamma: \Lambda]+1} \alpha \widetilde{f}\right]_{\Lambda}\right)=[\lambda \alpha \widetilde{f}]_{\Gamma}
$$

for mutual distinct lifting classes $\left[\lambda_{1} \alpha \widetilde{f}\right]_{\Lambda}, \ldots,\left[\lambda_{[\Gamma: \Lambda]+1} \alpha \widetilde{f}\right]_{\Lambda}$ of $\varphi(\alpha) \bar{f}$, where $\lambda_{1}, \ldots, \lambda_{[\Gamma: \Lambda]+1} \in \Lambda$. For $i=1, \ldots,[\Gamma: \Lambda]+1$, because the lifting classes $\left[\lambda_{i} \alpha \widetilde{f}\right]_{\Gamma}$ and $[\lambda \alpha \widetilde{f}]_{\Gamma}$ equal, there exists $\alpha_{i} \in \Gamma$ such that $\lambda_{i} \alpha \widetilde{f}=\alpha_{i} \lambda \alpha \widetilde{f} \alpha_{i}^{-1}$. By the pigeonhole principle, there exist $i, j$ such that $i \neq j$ but $\alpha_{i}=\mu \alpha_{j}$ for some $\mu \in \Lambda$. Then

$$
\lambda_{i} \alpha \widetilde{f}=\alpha_{i} \lambda \alpha \widetilde{f} \alpha_{i}^{-1}=\mu \alpha_{j} \lambda \alpha \widetilde{f} \alpha_{j}{ }^{-1} \mu^{-1}=\mu \lambda_{j} \alpha \widetilde{f} \mu^{-1},
$$

a contradiction with the fact that $\left[\lambda_{i} \alpha \widetilde{f}\right]_{\Lambda}$ and $\left[\lambda_{j} \alpha \widetilde{f}\right]_{\Lambda}$ are different lifting classes of $\varphi(\alpha) \bar{f}$. We see that $[\lambda \alpha \widetilde{f}]_{\Gamma}$ has at most $[\Gamma: \Lambda]$ preimages.

REMark 3.4. The implication $(a) \Rightarrow(b)$ is also stated for coincidences in [9, Theorem 2.4] in terms of the Lefschetz coincidence numbers of lifts to a nilcovering.

\section{Relation with Anosov diffeomorphisms}

In this section we prove that the Reidemeister number of an Anosov diffeomorphism on an infra-nilmanifold is always finite. Porteous [18] proves that the existence of an Anosov diffeomorphism on a closed flat manifold $M$ is determined by the holonomy representation of $M$. Let us first recall the definition of an Anosov diffeomorphism and a useful result.

Definition 4.1. A $C^{1}$ diffeomorphism $f: M \rightarrow M$ on a closed differentiable manifold $M$ is an Anosov diffeomorphism if and only if there exists a continuous $d f$-invariant splitting $T M=E^{s} \oplus E^{u}$ of the tangent bundle $T M$ of $M$ such that for any Riemannian metric $\|\cdot\|$ on $M$, there exist real constants $c>0$ and $\lambda \in(0,1)$ such that for all positive integers $n$ we have that

$$
\begin{array}{ll}
\left\|d f^{n}(v)\right\| \leq c \lambda^{n}\|v\| & \text { for all } v \in E^{s} \text { and } \\
\left\|d f^{n}(v)\right\| \geq c \lambda^{-n}\|v\| & \text { for all } v \in E^{u} .
\end{array}
$$

Lemma 4.2 ([15], [6, Lemma 2.1]). Let $f: M \rightarrow M$ be an Anosov diffeomorphism on an infra-nilmanifold $M$. Then $f$ has a homotopy lift $(d, D)$, where $D_{*}$ has no eigenvalue of modulus 1.

Proposition 4.3. Let $M$ be an infra-nilmanifold and suppose there exists a homeomorphism $f: M \rightarrow M$ with homotopy lift $(d, D)$ such that $D_{*}$ has no 
eigenvalue that is a root of unity. Then $R(f)<\infty$ and $M$ does not have the $R_{\infty}$ property.

ProOF. Let $\Gamma$ be the almost-Bieberbach group associated to $M$. We may assume that $f$ is induced by the homotopy lift $(d, D)$.

Suppose for a contradiction that $R(f)=\infty$. Then by Theorem 3.3, there exists $A$ in the holonomy group $F$ such that $(A D)_{*}$ has eigenvalue 1 . Because $f$ is a homeomorphism, $(d, D)$ belongs to the normaliser $N_{\mathrm{Aff}(G)}(\Gamma)$ of $\Gamma$ in $\operatorname{Aff}(G)$. Hence $D \in N_{\mathrm{Aut}(G)}(F)$. Now the centraliser $C_{\mathrm{Aut}(G)}(F)$ is a normal subgroup of the normaliser $N_{\mathrm{Aut}(G)}(F)$ and the quotient is isomorphic to a subgroup of $\operatorname{Aut}(F)$. Because $\operatorname{Aut}(F)$ is finite, there exists $k \in \mathbb{N} \backslash\{0\}$ such that $D^{k} \in$ $C_{\text {Aut }(G)}(F)$. There exists $A^{\prime} \in F$ such that $(A D)^{k}=A^{\prime} D^{k}$. Now pick $P \in$ $\mathrm{GL}_{n}(\mathbb{C})$ such that $P A_{*}^{\prime} P^{-1}$ and $P D_{*}^{k} P^{-1}$ are upper triangular matrices. Because $\left((A D)_{*}\right)^{k}=A_{*}^{\prime} D_{*}^{k}$ has eigenvalue 1 , there exists an eigenvalue $\lambda$ of $A_{*}^{\prime}$ and an eigenvalue $\mu$ of $D_{*}^{k}$ such that $\lambda \mu=1$. Because $A_{*}^{\prime}$ is a torsion element in $\operatorname{GL}_{n}(\mathbb{R})$, $\lambda$ is a root of unity and hence also $\mu$ is a root of unity. We see that $D_{*}^{k}$ and hence also $D_{*}$ has an eigenvalue that is a root of unity, a contradiction.

Corollary 4.4. Let $M$ be an infra-nilmanifold and suppose there exists an Anosov diffeomorphism $f: M \rightarrow M$. Then $R(f)<\infty$ and $M$ does not have the $R_{\infty}$ property

This corollary suggests studying the $R_{\infty}$ property for a closed flat manifold at the level of the holonomy representation. Indeed, Porteous [18] proves the following theorem.

THEOREM 4.5. Let $M$ be a closed flat manifold with holonomy representation $\rho: F \rightarrow \mathrm{GL}_{n}(\mathbb{Z})$. Then the following statements are equivalent:

(a) There exists an Anosov diffeomorphism on $M$.

(b) Each $\mathbb{Q}$-irreducible component of $\rho$ that occurs with multiplicity one is reducible over $\mathbb{R}$.

If a closed flat manifold $M$ has the $R_{\infty}$ property, the holonomy representation must have a $\mathbb{Q}$-irreducible component that is irreducible over $\mathbb{R}$ and occurs with multiplicity one. The converse does not need to hold, as is illustrated in the following example.

ExAMPLE 4.6. Let $M$ be the closed flat manifold of which the corresponding Bieberbach group is generated by $\mathbb{Z}^{4}$ and

$$
\left(\left(\begin{array}{c}
0 \\
0 \\
1 / 3 \\
0
\end{array}\right),\left(\begin{array}{cccc}
0 & 1 & 0 & 0 \\
-1 & -1 & 0 & 0 \\
0 & 0 & 1 & 0 \\
0 & 0 & 0 & 1
\end{array}\right)\right)
$$


Then the invertible affine endomorphism

$$
\left(\left(\begin{array}{l}
0 \\
0 \\
0 \\
0
\end{array}\right),\left(\begin{array}{cccc}
1 & 1 & 0 & 0 \\
-1 & 0 & 0 & 0 \\
0 & 0 & 13 & 21 \\
0 & 0 & 21 & 34
\end{array}\right)\right) \in \operatorname{Aff}\left(\mathbb{R}^{4}\right)
$$

induces a homeomorphism $f: M \rightarrow M$ of which the Reidemeister number $R(f)$ is finite.

Nevertheless, we have the following theorem.

THEOREM 4.7. Let $M$ be a closed flat manifold with holonomy representation $\rho: F \rightarrow \mathrm{GL}_{n}(\mathbb{Z})$. Let $\rho^{\prime}: F \rightarrow \mathrm{GL}_{n^{\prime}}(\mathbb{Z})$ be a $\mathbb{Q}$-irreducible $\mathbb{Z}$-subrepresentation of $\rho$ such that $\rho^{\prime}(F)$ is not $\mathbb{Q}$-conjugated to $\widetilde{\rho}(F)$ for any other $\mathbb{Q}$-subrepresentation $\widetilde{\rho}$ of $\rho$. Suppose moreover, that for every $D^{\prime} \in N_{\mathrm{GL}_{n^{\prime}}(\mathbb{Z})}\left(\rho^{\prime}(F)\right)$, there exists $A \in F$ such that $\rho^{\prime}(A) D^{\prime}$ has eigenvalue 1 . Then $M$ has the $R_{\infty}$ property.

By a $\mathbb{Z}$-subrepresentation $\rho^{\prime}$ of $\rho$, we mean a representation $\rho^{\prime}: F \rightarrow \mathrm{GL}_{n^{\prime}}(\mathbb{Z})$ such that there exist $P \in \mathrm{GL}_{n}(\mathbb{Z})$ and a representation $\rho^{\prime \prime}: F \rightarrow \mathrm{GL}_{n-n^{\prime}}(\mathbb{Z})$ such that for each $x \in F$, the matrix $P \rho(x) P^{-1}$ has the form of a blockmatrix

$$
P \rho(x) P^{-1}=\left(\begin{array}{cc}
\rho^{\prime}(x) & * \\
0 & \rho^{\prime \prime}(x)
\end{array}\right) .
$$

The necessity of the condition that for each $D^{\prime} \in N_{\mathrm{GL}_{n^{\prime}}(\mathbb{Z})}\left(\rho^{\prime}(F)\right)$, there exists $A \in F$ such that $\operatorname{det}\left(\mathbb{1}-\rho^{\prime}(A) D^{\prime}\right)=0$ is illustrated in Example 4.6. The necessity of the uniqueness condition on the image of the subrepresentation $\rho^{\prime}$ will be illustrated in Example 5.10, where we prove that the classical Hantzsche-Wendt manifold does not have the $R_{\infty}$ property. Remark that every $\mathbb{Q}$-irreducible component of the holonomy representation of the classical Hantzsche-Wendt manifold is irreducible over $\mathbb{R}$ and occurs with multiplicity 1.

Proof of Theorem 4.7. Let $\Gamma$ be the Bieberbach group associated to $M$. Without loss of generality, we may assume that $\Gamma \cap \mathbb{R}^{n}=\mathbb{Z}^{n}$. Let $f: M \rightarrow M$ be a homeomorphism. We want to prove that $R(f)=\infty$. Without loss of generality, we may assume that $f$ is induced by a homotopy lift $(d, D) \in \operatorname{Aff}\left(\mathbb{R}^{n}\right)$. Identifying $D_{*}$ with $D$, we have that $D \in N_{\mathrm{GL}_{n}(\mathbb{R})}(\rho(F))$. Because $(d, D)(z, \mathbb{1})(d, D)^{-1} \in \mathbb{Z}^{n}$ and $(d, D)^{-1}(z, \mathbb{1})(d, D) \in \mathbb{Z}^{n}$ for all $z \in \mathbb{Z}^{n}$, we have that $D \in \mathrm{GL}_{n}(\mathbb{Z})$. Hence $D \in N_{\mathrm{GL}_{n}(\mathbb{Z})}(\rho(F))$. Now there exist $\mathbb{Q}$-irreducible representations $\rho_{i}: F \rightarrow$ $\mathrm{GL}_{n_{i}}(\mathbb{Q})$ such that $\rho$ is similar to $\rho_{1} \oplus \ldots \oplus \rho_{t}$. We may assume that $\rho_{1}=\rho^{\prime}$. Write $\widehat{n}=n-n^{\prime}$. Now there exists $P_{1} \in \mathrm{GL}_{n}(\mathbb{Z})$ such that for all $A \in F$, the matrix $P_{1} \rho(A) P_{1}^{-1}$ is an upper triangular blockmatrix

$$
P_{1} \rho(A) P_{1}^{-1}=\left(\begin{array}{cc}
\rho^{\prime}(A) & * \\
0 & \widehat{\rho}(A)
\end{array}\right),
$$


where $\widehat{\rho}: F \rightarrow \mathrm{GL}_{\widehat{n}}(\mathbb{Z})$ is similar (over $\mathbb{Q}$ ) to $\rho_{2} \oplus \ldots \oplus \rho_{t}$ : there exists a matrix $\widehat{P} \in \mathrm{GL}_{\widehat{n}}(\mathbb{Q})$ such that

$$
\widehat{P} \widehat{\rho}(A) \widehat{P}^{-1}=\left(\rho_{2} \oplus \ldots \oplus \rho_{t}\right)(A),
$$

for all $A \in F$. Let $P_{2} \in \mathrm{GL}_{n}(\mathbb{Q})$ be the blockmatrix

$$
P_{2}=\left(\begin{array}{cc}
\mathbb{1}_{n^{\prime}} & 0 \\
0 & \widehat{P}
\end{array}\right) .
$$

Then for all $A \in F$, the matrix $P_{2} P_{1} \rho(A) P_{1}^{-1} P_{2}^{-1}$ has the form

$$
P_{2} P_{1} \rho(A) P_{1}^{-1} P_{2}^{-1}=\left(\begin{array}{cccc}
\rho_{1}(A) & * & \ldots & * \\
& \ddots & \ddots & \vdots \\
& & \ddots & * \\
& & & \rho_{t}(A)
\end{array}\right) .
$$

Now it is well known that we can find a blockmatrix

$$
P_{3}=\left(\begin{array}{cccc}
\mathbb{1}_{n_{1}} & * & \cdots & * \\
& \ddots & \ddots & \vdots \\
& & \ddots & * \\
& & & \mathbb{1}_{n_{t}}
\end{array}\right) \in \mathrm{GL}_{n}(\mathbb{Q})
$$

such that, for all $A \in F$,

$$
P_{3}\left(\begin{array}{cccc}
\rho_{1}(A) & * & \ldots & * \\
& \ddots & \ddots & \vdots \\
& & \ddots & * \\
& & & \rho_{t}(A)
\end{array}\right) P_{3}^{-1}=\left(\rho_{1} \oplus \ldots \oplus \rho_{t}\right)(A) .
$$

Put $P=P_{3} P_{2} P_{1}$. Write $P D P^{-1}$ as a blockmatrix

$$
P D P^{-1}=\left(\begin{array}{ccc}
D_{1,1} & \ldots & D_{1, t} \\
\vdots & & \vdots \\
D_{t, 1} & \ldots & D_{t, t}
\end{array}\right) \quad \text { with } D_{i, j} \in \mathbb{Q}^{n_{i} \times n_{j}} .
$$

For every $A \in F$, there exists $\phi(A) \in F$ such that $\rho(\phi(A)) D=D \rho(A)$. Now the map $\phi: F \rightarrow F$ is an automorphism. Choose $i \in\{2, \ldots, t\}$. Remark that $\rho_{i}(\phi(A)) D_{i, 1}=D_{i, 1} \rho^{\prime}(A)$ for all $A \in F$. Hence $\operatorname{Ker}\left(D_{i, 1}\right)$ is invariant under $\rho^{\prime}$ and because $\phi$ is an automorphism, $\operatorname{Im}\left(D_{i, 1}\right)$ is invariant under $\rho_{i}$.

We want to prove that $D_{i, 1}=0$. We consider three cases, according to whether $n_{i}<n^{\prime}, n_{i}=n^{\prime}$ or $n_{i}>n^{\prime}$.

Suppose $n_{i}<n^{\prime}$. In this case, because $D_{i, 1} \in \mathbb{Q}^{n_{i} \times n^{\prime}}$, we must have that $\operatorname{Ker}\left(D_{i, 1}\right) \neq\{0\}$. Because $\operatorname{Ker}\left(D_{i, 1}\right)$ is invariant under $\rho^{\prime}$, this implies that $D_{i, 1}=0$.

Now consider the case $n_{i}=n^{\prime}$. Suppose for a contradiction that $D_{i, 1} \neq 0$. 
Because $\operatorname{Ker}\left(D_{i, 1}\right)$ is invariant under $\rho^{\prime}$, this implies that $\operatorname{Ker}\left(D_{i, 1}\right)=\{0\}$. Because $D_{i, 1}$ is a square matrix, $\rho^{\prime}(A)=D_{i, 1}^{-1} \rho_{i}(\phi(A)) D_{i, 1}$ for all $A \in F$. This is a contradiction with the assumption that $\rho^{\prime}(F)$ is not conjugated to $\rho_{i}(F)$. We see that $D_{i, 1}=0$ when $n_{i}=n^{\prime}$.

In the case $n_{i}>n^{\prime}$, we have that $\operatorname{Im}\left(D_{i, 1}\right) \neq \mathbb{Q}^{n_{i}}$ because $D_{i, 1} \in \mathbb{Q}^{n_{i} \times n^{\prime}}$. Because $\operatorname{Im}\left(D_{i, 1}\right)$ is invariant under $\rho_{i}$, we have that $D_{i, 1}=0$.

We conclude that in each of the three cases, $D_{i, 1}=0$. Thus $P_{1} D P_{1}^{-1}=$ $P_{2}^{-1} P_{3}^{-1} P D P^{-1} P_{3} P_{2}$ has the form

$$
P_{1} D P_{1}^{-1}=\left(\begin{array}{cc}
D^{\prime} & * \\
0 & \widehat{D}
\end{array}\right) \in \mathrm{GL}_{n}(\mathbb{Z})
$$

where $D^{\prime} \in \mathrm{GL}_{n^{\prime}}(\mathbb{Z})$ and $\widehat{D} \in \mathrm{GL}_{\widehat{n}}(\mathbb{Z})$. Moreover, $D^{\prime} \in N_{\mathrm{GL}_{n^{\prime}}(\mathbb{Z})}\left(\rho^{\prime}(F)\right)$. Hence there exists $A \in F$ such that $\rho^{\prime}(A) D^{\prime}$ has eigenvalue 1 . Then also $\rho(A) D$ has eigenvalue 1. By Theorem 3.3, $R(f)=\infty$.

We finish this section by formulating a conjecture:

CONJECTURe 4.8. Let $\rho: F \rightarrow \mathrm{GL}_{n}(\mathbb{Z})$ be a faithful $\mathbb{R}$-irreducible representation of a non-trivial finite group $F$. Suppose that $n$ is odd. Then for every $D \in N_{\mathrm{GL}_{n}(\mathbb{Z})}(\rho(F))$, there exists $A \in F$ such that $\rho(A) D$ has eigenvalue 1 .

We have checked this conjecture by computer for $n=1,3,5$. The following example illustrates the necessity of the condition that $n$ is odd, even if we require that $\rho$ is irreducible over $\mathbb{C}$.

ExAmPLE 4.9. Let $F$ be the finite subgroup of $\mathrm{GL}_{4}(\mathbb{Z})$ generated by

$$
\left(\begin{array}{cccc}
0 & 0 & -1 & 0 \\
1 & 1 & 1 & -2 \\
-1 & 0 & 0 & 0 \\
0 & 0 & 0 & -1
\end{array}\right), \quad\left(\begin{array}{cccc}
1 & 0 & 0 & 0 \\
0 & -1 & 0 & 0 \\
0 & 0 & 1 & 0 \\
1 & 0 & 1 & -1
\end{array}\right), \quad\left(\begin{array}{cccc}
1 & 0 & 0 & 0 \\
0 & -1 & 0 & 0 \\
0 & 0 & -1 & 0 \\
0 & -1 & -1 & 1
\end{array}\right), \quad\left(\begin{array}{cccc}
-1 & -1 & -1 & 2 \\
0 & 0 & 1 & 0 \\
0 & -1 & 0 & 0 \\
-1 & -1 & 0 & 1
\end{array}\right)
$$

and let $\rho: F \rightarrow \mathrm{GL}_{4}(\mathbb{Z})$ be the inclusion representation. Then $\rho$ is irreducible over $\mathbb{C}$. Put

$$
D=\left(\begin{array}{cccc}
-1 & -1 & -1 & 1 \\
-1 & 0 & 0 & 1 \\
0 & 0 & -1 & 1 \\
-1 & -1 & -1 & 2
\end{array}\right)
$$

Then $D \in N_{\mathrm{GL}_{4}(\mathbb{Z})}(\rho(F))$, but there exists no $A \in F$ such that $\rho(A) D$ has eigenvalue 1 .

\section{The $R_{\infty}$ property for GHW manifolds}

In this section, we prove that a non-orientable GHW manifold has the $R_{\infty}$ property and that an HW manifold does not have the $R_{\infty}$ property if and only if it is homeomorphic to an HW manifold in standard form of which the associated graph is circulant. Recall that a graph is circulant if and only if it has an 
automorphism which is, considered as a permutation of the vertices, a cycle of full length.

In order to prove this result, we first need to prove a series of technical lemmas.

Definition 5.1. Let $\sigma \in S_{n}$ be a permutation. Then define $P_{\sigma}$ as the matrix with $e_{\sigma(i)}$ as the $i$-th column, where $e_{\sigma(i)} \in \mathbb{Z}^{n}$ is the vector having 1 on the $\sigma(i)$-th place and 0 elsewhere.

REMARK 5.2. Let $\sigma \in S_{n}$ be a permutation and $v={ }^{t}\left(v_{1}, \ldots, v_{n}\right) \in \mathbb{R}^{n}$. Then

$$
P_{\sigma} v={ }^{t}\left(v_{\sigma^{-1}(1)}, \ldots, v_{\sigma^{-1}(n)}\right) .
$$

Definition 5.3. Let $\sigma \in S_{n}$ be a permutation and $a_{1}, \ldots, a_{n} \in \mathbb{R}$. Then define

$$
M_{\sigma}\left(a_{1}, \ldots, a_{n}\right)=P_{\sigma} \operatorname{diag}\left(a_{1}, \ldots, a_{n}\right) .
$$

Remark 5.4. Let $\sigma \in S_{n}$ be a permutation and $a_{1}, \ldots, a_{n} \in \mathbb{R}$. Then

$$
P_{\sigma} \operatorname{diag}\left(a_{1}, \ldots, a_{n}\right)=\operatorname{diag}\left(a_{\sigma^{-1}(1)}, \ldots, a_{\sigma^{-1}(n)}\right) P_{\sigma} .
$$

The characteristic polynomial of $M_{\sigma}\left(a_{1}, \ldots, a_{n}\right)$ has a special form when $\sigma$ is the permutation $(1 \ldots n)$.

Lemma 5.5. Let $\sigma \in S_{n}$ be the permutation $(1 \ldots n)$. Let $a_{1}, \ldots, a_{n} \in \mathbb{R}$. Then for all $\lambda \in \mathbb{C}$,

$$
\operatorname{det}\left(\lambda \mathbb{1}_{n}-M_{\sigma}\left(a_{1}, \ldots, a_{n}\right)\right)=\lambda^{n}-a_{1} \ldots a_{n} .
$$

This lemma is easy to prove since the first row of $\lambda \mathbb{1}_{n}-M_{\sigma}\left(a_{1}, \ldots, a_{n}\right)$ has at most two non-zero entries.

The following proposition is a generalisation of [5, Proposition 3.3(2.)].

Proposition 5.6. Let $M$ be a GHW manifold with associated GHW group $\Gamma$ and holonomy representation $\rho: \mathbb{Z}_{2}^{n-1} \rightarrow \mathrm{GL}_{n}(\mathbb{Z})$. Suppose that $\Gamma \cap \mathbb{R}^{n}=\mathbb{Z}^{n}$ and that $\rho(x)$ is a diagonal matrix for all $x \in \mathbb{Z}_{2}^{n-1}$. Let $f: M \rightarrow M$ be a homeomorphism and suppose that $f$ has a lift $(d, D) \in \operatorname{Aff}\left(\mathbb{R}^{n}\right)$. Then there exists a permutation $\sigma \in S_{n}$ and $\varepsilon_{1}, \ldots, \varepsilon_{n} \in\{-1,1\}$ such that $D=M_{\sigma}\left(\varepsilon_{1}, \ldots, \varepsilon_{n}\right)$.

Proof. Write $\rho=\rho_{1} \oplus \ldots \oplus \rho_{n}$, with $\rho_{i}: \mathbb{Z}_{2}^{n-1} \rightarrow \mathrm{GL}_{1}(\mathbb{Z})$. Suppose $\rho_{i}=\rho_{j}$, but $i \neq j$. Then $\rho_{1} \oplus \ldots \oplus \rho_{j-1} \oplus \rho_{j+1} \oplus \ldots \oplus \rho_{n}: \mathbb{Z}_{2}^{n-1} \rightarrow \mathrm{GL}_{n-1}(\mathbb{Z})$ is a faithful representation. Hence the image has order $2^{n-1}$ and contains -1 . Then also $\rho\left(\mathbb{Z}_{2}^{n-1}\right)$ contains $-\mathbb{1}$ and $\Gamma$ contains a torsion element $(a,-\mathbb{1})$, a contradiction. We conclude that $\rho_{i} \neq \rho_{j}$ when $i \neq j$. For every $x \in \mathbb{Z}_{2}^{n-1}$, there exists $\phi(x) \in$ $\mathbb{Z}_{2}^{n-1}$ such that $\rho(\phi(x)) D=D \rho(x)$. Write $D=\left(d_{i, j}\right)_{i, j}$, then

$$
\rho_{i}(\phi(x)) d_{i, j}=d_{i, j} \rho_{j}(x) \text { for all } x \in \mathbb{Z}_{2}^{n-1} .
$$


Because $\operatorname{det}(D) \neq 0$, for each $i$ there exists a $j$ such that $d_{i, j} \neq 0$.

We now prove that for each $i$, exactly one $j$ exists such that $d_{i, j} \neq 0$. Pick $i \in$ $\{1, \ldots, n\}$ and suppose that $d_{i, j_{1}} \neq 0$ and $d_{i, j_{2}} \neq 0$ for some $j_{1}, j_{2} \in\{1, \ldots, n\}$. Because

$$
\rho_{i}(\phi(x)) d_{i, j_{1}}=d_{i, j_{1}} \rho_{j_{1}}(x) \quad \text { and } \quad \rho_{i}(\phi(x)) d_{i, j_{2}}=d_{i, j_{2}} \rho_{j_{2}}(x)
$$

for all $x \in \mathbb{Z}_{2}^{n-1}$, we have that $\rho_{j_{1}}(x)=\rho_{j_{2}}(x)$ for all $x \in \mathbb{Z}_{2}^{n-1}$, and hence $j_{1}=j_{2}$.

We see that for each $i$, there exists exactly one $\psi(i)$ such that $d_{i, \psi(i)} \neq 0$. Then $\psi:\{1, \ldots, n\} \rightarrow\{1, \ldots, n\}: i \mapsto \psi(i)$ is a function. Because $\operatorname{det}(D) \neq 0$, for each $j$, there exists $i$ such that $d_{i, j} \neq 0$. Hence $\psi$ is a bijection. Put $\sigma=\psi^{-1}$. Define $\varepsilon_{1}, \ldots, \varepsilon_{n}$ by $\varepsilon_{j}=d_{\sigma(j), j}$, then $D=M_{\sigma}\left(\varepsilon_{1}, \ldots, \varepsilon_{n}\right)$.

Because $(d, D)(z, \mathbb{1})(d, D)^{-1} \in \mathbb{Z}^{n}$ and $(d, D)^{-1}(z, \mathbb{1})(d, D) \in \mathbb{Z}^{n}$ for each $z \in \mathbb{Z}^{n}$, we have that $D \in \mathrm{GL}_{n}(\mathbb{Z})$, such that $\varepsilon_{i} \in \mathbb{Z}$. Because $\pm 1=\operatorname{det}(D)=$ $\operatorname{sign}(\sigma) \varepsilon_{1} \ldots \varepsilon_{n}$, we have that $\varepsilon_{i} \in\{-1,1\}$ for $i=1, \ldots, n$.

If $f$ is a self-homeomorphism on an HW manifold $M$ in standard form, the previous proposition tells us that the linear part $D$ of a homotopy lift $(d, D)$ of $f$ must have the form $M_{\sigma}\left(\varepsilon_{1}, \ldots, \varepsilon_{n}\right)$ for a permutation $\sigma$. The following proposition helps to determine the possibilities for this permutation $\sigma$.

Proposition 5.7. Let $\Gamma$ be an HW group in standard form. Let $\varepsilon_{1}, \ldots, \varepsilon_{n} \in$ $\{-1,1\}$ and let $\sigma \in S_{n}$ be a permutation such that $\sigma(i) \neq i$ for all $i \in\{1, \ldots, n\}$. Then there exists $d \in \mathbb{R}^{n}$ such that $\left(d, M_{\sigma}\left(\varepsilon_{1}, \ldots, \varepsilon_{n}\right)\right) \in N_{\mathrm{Aff}\left(\mathbb{R}^{n}\right)}(\Gamma)$ if and only if there exists $a \in \mathbb{R}^{n}$ such that $(a, \mathbb{1}) \Gamma(-a, \mathbb{1})$ is an $\mathrm{HW}$ group in standard form for which $\sigma$ is an automorphism of the graph associated to $(a, \mathbb{1}) \Gamma(-a, \mathbb{1})$.

Proof. Suppose there exists $a \in \mathbb{R}^{n}$ such that $(a, \mathbb{1}) \Gamma(-a, \mathbb{1})$ is an HW group in standard form for which $\sigma$ is an automorphism of the graph associated to $(a, \mathbb{1}) \Gamma(-a, \mathbb{1})$. Then for $i=1, \ldots, n$, there exist $b_{i}=\left(b_{i, 1}, \ldots, b_{i, n}\right) \in$ $\{0,1 / 2\}^{n}$ with $b_{i, i}=1 / 2$ such that $(a, \mathbb{1}) \Gamma(-a, \mathbb{1})$ is generated by $\mathbb{Z}^{n}$ and $\left(b_{1}, B_{1}\right), \ldots,\left(b_{n}, B_{n}\right)$, where $B_{i}$ is the diagonal matrix with 1 on the $i$-th place of the diagonal and -1 on the other places of the diagonal. Without loss of generality, we may assume that $\sigma$ is an automorphism of the graph associated to $\Gamma$. Indeed, when $\left(d, M_{\sigma}\left(\varepsilon_{1}, \ldots, \varepsilon_{n}\right)\right) \in N_{\mathrm{Aff}\left(\mathbb{R}^{n}\right)}((a, \mathbb{1}) \Gamma(-a, \mathbb{1}))$, we have that $(-a, \mathbb{1})\left(d, M_{\sigma}\left(\varepsilon_{1}, \ldots, \varepsilon_{n}\right)\right)(a, \mathbb{1}) \in N_{\mathrm{Aff}\left(\mathbb{R}^{n}\right)}(\Gamma)$.

Suppose that $\sigma$ is an automorphism of the graph associated to $\Gamma$. Put $d=$ $0 \in \mathbb{R}^{n}$. Define $\left(b_{1}^{\prime}, B_{1}^{\prime}\right), \ldots,\left(b_{n}^{\prime}, B_{n}^{\prime}\right)$ by

$$
\left(b_{\sigma(i)}^{\prime}, B_{\sigma(i)}^{\prime}\right)=\left(d, M_{\sigma}\left(\varepsilon_{1}, \ldots, \varepsilon_{n}\right)\right)\left(b_{i}, B_{i}\right)\left(d, M_{\sigma}\left(\varepsilon_{1}, \ldots, \varepsilon_{n}\right)\right)^{-1}
$$

for $i=1, \ldots, n$. Then it suffices to show that $\left(b_{\sigma(i)}^{\prime}, B_{\sigma(i)}^{\prime}\right) \in \Gamma$ for $i=1, \ldots, n$. 
Choose an arbitrary $i \in\{1, \ldots, n\}$. Then

$$
\begin{aligned}
\left(b_{\sigma(i)}^{\prime}, B_{\sigma(i)}^{\prime}\right) & =\left(0, M_{\sigma}\left(\varepsilon_{1}, \ldots, \varepsilon_{n}\right)\right)\left(b_{i}, B_{i}\right)\left(0, M_{\sigma}\left(\varepsilon_{1}, \ldots, \varepsilon_{n}\right)\right)^{-1} \\
& =\left(M_{\sigma}\left(\varepsilon_{1}, \ldots, \varepsilon_{n}\right) b_{i}, M_{\sigma}\left(\varepsilon_{1}, \ldots, \varepsilon_{n}\right) B_{i}\right)\left(0, M_{\sigma}\left(\varepsilon_{1}, \ldots, \varepsilon_{n}\right)^{-1}\right) \\
& =\left(M_{\sigma}\left(\varepsilon_{1}, \ldots, \varepsilon_{n}\right) b_{i}, M_{\sigma}\left(\varepsilon_{1}, \ldots, \varepsilon_{n}\right) B_{i} M_{\sigma}\left(\varepsilon_{1}, \ldots, \varepsilon_{n}\right)^{-1}\right) \\
& =\left(M_{\sigma}\left(\varepsilon_{1}, \ldots, \varepsilon_{n}\right) b_{i}, B_{\sigma(i)}\right)
\end{aligned}
$$

such that $B_{\sigma(i)}^{\prime}=B_{\sigma(i)}$. Because $\sigma$ is an automorphism of the graph associated to $\Gamma$, we have that $b_{i, \sigma^{-1}(j)}=b_{\sigma(i), j}$ for $j=1, \ldots, n$. Hence

$$
\begin{aligned}
b_{\sigma(i)}^{\prime} & =M_{\sigma}\left(\varepsilon_{1}, \ldots, \varepsilon_{n}\right) b_{i} \\
& =P_{\sigma} \operatorname{diag}\left(\varepsilon_{1}, \ldots, \varepsilon_{n}\right)^{t}\left(b_{i, 1}, \ldots, b_{i, n}\right)=P_{\sigma}{ }^{t}\left(\varepsilon_{1} b_{i, 1}, \ldots, \varepsilon_{n} b_{i, n}\right) \\
& ={ }^{t}\left(\varepsilon_{\sigma^{-1}(1)} b_{i, \sigma^{-1}(1)}, \ldots, \varepsilon_{\sigma^{-1}(n)} b_{i, \sigma^{-1}(n)}\right) \\
& ={ }^{t}\left(\varepsilon_{\sigma^{-1}(1)} b_{\sigma(i), 1}, \ldots, \varepsilon_{\sigma^{-1}(n)} b_{\sigma(i), n}\right) .
\end{aligned}
$$

Define $z_{i, 1}, \ldots, z_{i, n}$ by

$$
z_{i, j}=\left\{\begin{array}{ll}
1 & \text { if } \varepsilon_{\sigma^{-1}(j)} b_{\sigma(i), j}=-1 / 2, \\
0 & \text { otherwise }
\end{array} \text { for } j=1, \ldots, n .\right.
$$

Put $z_{i}=\left(z_{i, 1}, \ldots, z_{i, n}\right)$, then $b_{\sigma(i)}^{\prime}=b_{\sigma(i)}-z_{i}$. Hence $\left(b_{\sigma(i)}^{\prime}, B_{\sigma(i)}^{\prime}\right)=\left(b_{\sigma(i)}-\right.$ $\left.z_{i}, B_{\sigma(i)}\right) \in \Gamma$.

Conversely, suppose there exists a vector $d=\left(d_{1}, \ldots, d_{n}\right) \in \mathbb{R}^{n}$ such that $\left(d, M_{\sigma}\left(\varepsilon_{1}, \ldots, \varepsilon_{n}\right)\right) \in N_{\mathrm{Aff}\left(\mathbb{R}^{n}\right)}(\Gamma)$. Because $\Gamma$ is in standard form, for $i=$ $1, \ldots, n$, there exist $b_{i}=\left(b_{i, 1}, \ldots, b_{i, n}\right) \in\{0,1 / 2\}^{n}$ with $b_{i, i}=1 / 2$ such that $\Gamma$ is generated by $\mathbb{Z}^{n}$ and $\left(b_{1}, B_{1}\right), \ldots,\left(b_{n}, B_{n}\right)$, where $B_{i}$ is the diagonal matrix with 1 on the $i$-th place of the diagonal and -1 on the other places of the diagonal.

Choose an arbitrary $i \in\{1, \ldots, n\}$. Define

$$
\left(b_{\sigma(i)}^{\prime}, B_{\sigma(i)}^{\prime}\right)=\left(d, M_{\sigma}\left(\varepsilon_{1}, \ldots, \varepsilon_{n}\right)\right)\left(b_{i}, B_{i}\right)\left(d, M_{\sigma}\left(\varepsilon_{1}, \ldots, \varepsilon_{n}\right)\right)^{-1} \in \Gamma,
$$

then

$$
\begin{aligned}
& \left(b_{\sigma(i)}^{\prime}+B_{\sigma(i)}^{\prime} d, B_{\sigma(i)}^{\prime} M_{\sigma}\left(\varepsilon_{1}, \ldots, \varepsilon_{n}\right)\right)=\left(b_{\sigma(i)}^{\prime}, B_{\sigma(i)}^{\prime}\right)\left(d, M_{\sigma}\left(\varepsilon_{1}, \ldots, \varepsilon_{n}\right)\right) \\
& \quad=\left(d, M_{\sigma}\left(\varepsilon_{1}, \ldots, \varepsilon_{n}\right)\right)\left(b_{i}, B_{i}\right)=\left(d+M_{\sigma}\left(\varepsilon_{1}, \ldots, \varepsilon_{n}\right) b_{i}, M_{\sigma}\left(\varepsilon_{1}, \ldots, \varepsilon_{n}\right) B_{i}\right) .
\end{aligned}
$$

Hence $B_{\sigma(i)}^{\prime}=M_{\sigma}\left(\varepsilon_{1}, \ldots, \varepsilon_{n}\right) B_{i} M_{\sigma}\left(\varepsilon_{1}, \ldots, \varepsilon_{n}\right)^{-1}=B_{\sigma(i)}$.

Because $\left(b_{\sigma(i)}^{\prime}, B_{\sigma(i)}\right) \in \Gamma$ and $\left(b_{\sigma(i)}, B_{\sigma(i)}\right) \in \Gamma$, there exists $z_{i} \in \mathbb{Z}^{n}$ such that $b_{\sigma(i)}^{\prime}=b_{\sigma(i)}+z_{i}$. Thus $b_{\sigma(i)}+z_{i}+B_{\sigma(i)} d=d+M_{\sigma}\left(\varepsilon_{1}, \ldots, \varepsilon_{n}\right) b_{i}$, and hence

$$
b_{\sigma(i)}-M_{\sigma}\left(\varepsilon_{1}, \ldots, \varepsilon_{n}\right) b_{i}+\left(B_{\sigma(i)}-\mathbb{1}\right) d=-z_{i} \in \mathbb{Z}^{n} .
$$

Choose $j \in\{1, \ldots, n\} \backslash\{\sigma(i)\}$. Then

$$
b_{\sigma(i), j}-\varepsilon_{\sigma^{-1}(j)} b_{i, \sigma^{-1}(j)}-2 d_{j} \in \mathbb{Z} .
$$


If $2 d_{j} \in \mathbb{Z}$, then $b_{\sigma(i), j}=b_{i, \sigma^{-1}(j)}$. If $2 d_{j} \notin \mathbb{Z}$ then $b_{\sigma(i), j} \neq b_{i, \sigma^{-1}(j)}$. We conclude that

$$
b_{\sigma(i), j}=b_{i, \sigma^{-1}(j)} \Leftrightarrow 2 d_{j} \in \mathbb{Z} \quad \text { for all } i, j \text { where } j \neq \sigma(i) .
$$

We now use induction on $\#\left\{j \mid b_{\sigma(j), j}=0\right\}$ to prove there exists $a \in \mathbb{R}^{n}$ such that $(a, \mathbb{1}) \Gamma(-a, \mathbb{1})$ is an HW group in standard form such that $\sigma$ is an automorphism of the graph associated to $(a, \mathbb{1}) \Gamma(-a, \mathbb{1})$.

Let us first prove the induction basis. Suppose that $\#\left\{j \mid b_{\sigma(j), j}=0\right\}=0$. Because of the assumption that $b_{i, j} \in\{0,1 / 2\}$ for all $i, j$, we have that $b_{\sigma(j), j}=$ $1 / 2$ for all $j \in\{1, \ldots, n\}$. For every $j \in\{1, \ldots, n\}$ we have that $j \neq \sigma(j)$ and $b_{\sigma(j), j}=1 / 2=b_{j, \sigma^{-1}(j)}$, such that by (5.1) we conclude that $2 d_{j} \in \mathbb{Z}$. Hence also $2 d_{\sigma(j)} \in \mathbb{Z}$ for any $j$. By (5.1), we have that $b_{\sigma(i), \sigma(j)}=b_{i, j}$ for all $i \neq j$. Because also $b_{\sigma(j), \sigma(j)}=1 / 2=b_{j, j}$, we have that $b_{\sigma(i), \sigma(j)}=b_{i, j}$ for all $i, j$. We see that $\sigma$ is an automorphism of the graph associated to $\Gamma$. Hence we may put $a=0 \in \mathbb{R}^{n}$.

Let us now prove the induction step. Suppose that $\#\left\{j \mid b_{\sigma(j), j}=0\right\}>0$.

Pick $j_{1}$ such that $b_{\sigma\left(j_{1}\right), j_{1}}=0$. Let $a^{\prime} \in \mathbb{R}^{n}$ be the vector with $1 / 4$ on the $j_{1}$-th place and zero elsewhere.

Define $\widetilde{\Gamma}=\left(a^{\prime}, \mathbb{1}\right) \Gamma\left(-a^{\prime}, \mathbb{1}\right)$. Define $\left(\widehat{b}_{1}, \widehat{B}_{1}\right), \ldots,\left(\widehat{b}_{n}, \widehat{B}_{n}\right)$ by

$$
\left(\widehat{b}_{i}, \widehat{B}_{i}\right)=\left(a^{\prime}, \mathbb{1}\right)\left(b_{i}, B_{i}\right)\left(-a^{\prime}, \mathbb{1}\right) \text { for } i=1, \ldots, n,
$$

then

$$
\left(\widehat{b}_{i}, \widehat{B}_{i}\right)=\left(a^{\prime}+b_{i}-B_{i} a^{\prime}, B_{i}\right) \text { for } i=1, \ldots, n \text {. }
$$

We see that $\widehat{B}_{1}=B_{1}, \ldots, \widehat{B}_{n}=B_{n}$. Additionally, $\widehat{b}_{j_{1}}=b_{j_{1}}$ and $\widehat{b}_{j}=b_{j}+2 a^{\prime}$ when $j \neq j_{1}$.

Choose an arbitrary $i \in\{1, \ldots, n\}$. Write $\widehat{b}_{i}=\left(\widehat{b}_{i, 1}, \ldots, \widehat{b}_{i, n}\right)$, then $\widehat{b}_{i, j} \in \frac{1}{2} \mathbb{Z}$ for all $j$. Now choose $z_{i} \in \mathbb{Z}^{n}$ such that $\widehat{b}_{i}+z_{i} \in\{0,1 / 2\}^{n}$. Put $\widetilde{b}_{i}=\widehat{b}_{i}+z_{i}$ and write $\widetilde{b}_{i}=\left(\widetilde{b}_{i, 1}, \ldots, \widetilde{b}_{i, n}\right)$. Then $\widetilde{\Gamma}$ is generated by $\mathbb{Z}^{n}$ and $\left(\widetilde{b}_{1}, B_{1}\right), \ldots,\left(\widetilde{b}_{n}, B_{n}\right)$, where $\widetilde{b}_{i} \in\{0,1 / 2\}^{n}$.

We see that $\widetilde{\Gamma}$ is an HW group in standard form. Now for all $j \neq j_{1}$, the vector $a^{\prime}$ has a 0 on the $j$-th place and hence $\widehat{b}_{\sigma(j), j}=b_{\sigma(j), j}+0$ such that $\widetilde{b}_{\sigma(j), j}=b_{\sigma(j), j}$. Because $\widehat{b}_{\sigma\left(j_{1}\right), j_{1}}=b_{\sigma\left(j_{1}\right), j_{1}}+1 / 2=1 / 2$, we have that $\widetilde{b}_{\sigma\left(j_{1}\right), j_{1}}=1 / 2$. Hence $\#\left\{j \mid \widetilde{b}_{\sigma(j), j}=0\right\}=\#\left\{j \mid b_{\sigma(j), j}=0\right\}-1$. Put

$$
\left(\widetilde{d}, M_{\sigma}\left(\varepsilon_{1}, \ldots, \varepsilon_{n}\right)\right)=\left(a^{\prime}, \mathbb{1}\right)\left(d, M_{\sigma}\left(\varepsilon_{1}, \ldots, \varepsilon_{n}\right)\right)\left(-a^{\prime}, \mathbb{1}\right),
$$

then $\left(\widetilde{d}, M_{\sigma}\left(\varepsilon_{1}, \ldots, \varepsilon_{n}\right)\right) \in N_{\mathrm{Aff}\left(\mathbb{R}^{n}\right)}(\widetilde{\Gamma})$. Because $\#\left\{j \mid \widetilde{b}_{\sigma(j), j}=0\right\}=\#\{j \mid$ $\left.b_{\sigma(j), j}=0\right\}-1$, we may apply the induction hypothesis and conclude there exists $\widetilde{a} \in \mathbb{R}^{n}$ such that $(\widetilde{a}, \mathbb{1}) \widetilde{\Gamma}(-\widetilde{a}, \mathbb{1})$ is an HW group in standard form for which $\sigma$ is an automorphism of the graph associated to $(\widetilde{a}, \mathbb{1}) \widetilde{\Gamma}(-\widetilde{a}, \mathbb{1})$. 
Put $a=a^{\prime}+\widetilde{a}$, then $\sigma$ is an automorphism of the graph associated to $(a, \mathbb{1}) \Gamma(-a, \mathbb{1})$.

In Proposition 5.7, the condition that $\sigma(j) \neq j$ for all $j \in\{1, \ldots, n\}$ cannot be dropped, as is illustrated in the following example.

ExAmple 5.8. Let $M$ be the classical Hantzsche-Wendt manifold. The associated Bieberbach group $\Gamma$ is in standard form and is generated by $\mathbb{Z}^{3}$ and

$$
\left(\left(\begin{array}{c}
1 / 2 \\
0 \\
0
\end{array}\right),\left(\begin{array}{ccc}
1 & 0 & 0 \\
0 & -1 & 0 \\
0 & 0 & -1
\end{array}\right)\right) \text { and }\left(\left(\begin{array}{c}
0 \\
1 / 2 \\
1 / 2
\end{array}\right),\left(\begin{array}{ccc}
-1 & 0 & 0 \\
0 & 1 & 0 \\
0 & 0 & -1
\end{array}\right)\right) \text {. }
$$

The affine endomorphism

$$
\left(\left(\begin{array}{c}
1 / 4 \\
0 \\
0
\end{array}\right),\left(\begin{array}{lll}
1 & 0 & 0 \\
0 & 0 & 1 \\
0 & 1 & 0
\end{array}\right)\right)
$$

induces a homeomorphism $f: M \rightarrow M$, but one can check that the permutation (23) is no automorphism of any of the graphs $(\{1,2,3\}, E)$ that satisfy the conditions in Theorem 2.3.

Let us now state the main theorem of this section:

TheOREM 5.9. A non-orientable GHW manifold has the $R_{\infty}$ property. An HW manifold does not have the $R_{\infty}$ property if and only if it is homeomorphic to an HW manifold in standard form of which the associated graph is circulant.

Proof. Let $M$ be a GHW manifold with associated Bieberbach group $\Gamma$. Suppose $M$ is not orientable or not homeomorphic to an HW manifold in standard form of which the associated graph is circulant. We want to prove that $M$ has the $R_{\infty}$ property. In the case $M$ is orientable, we may suppose that $\Gamma$ is in standard form. In the case $M$ is not orientable, we may suppose that $\Gamma \cap \mathbb{R}^{n}=\mathbb{Z}^{n}$ and that $\rho(x)$ is a diagonal matrix for all $x \in \mathbb{Z}_{2}^{n-1}$. Let $f: M \rightarrow M$ be a homeomorphism with homotopy lift $(d, D)$. We want to prove that $R(f)=\infty$. By Proposition 5.6 we know that $D=M_{\sigma}\left(\varepsilon_{1}, \ldots, \varepsilon_{n}\right)$ for a permutation $\sigma \in S_{n}$ and $\varepsilon_{1}, \ldots, \varepsilon_{n} \in\{-1,1\}$. By permuting the elements of the standard basis of $\Gamma \cap \mathbb{R}^{n}=\mathbb{Z}^{n}$, if necessary, we may assume that $\sigma=\left(1 \ldots n_{1}\right)\left(n_{1}+1 \ldots n_{2}\right) \ldots\left(n_{t-1}+1 \ldots n_{t}\right)$ where $0=n_{0}<n_{1}<n_{2}<$ $\ldots<n_{t}=n$. Then $\rho(x)$ is still a diagonal matrix for all $x \in \mathbb{Z}_{2}^{n-1}$ and $\Gamma$ remains in standard form if it was. We can write $D$ as a blockmatrix

$$
D=\left(\begin{array}{ccc}
D_{1} & & 0 \\
& \ddots & \\
0 & & D_{t}
\end{array}\right)
$$


with $D_{i}=M_{\sigma_{i}}\left(\varepsilon_{i, 1}, \ldots, \varepsilon_{i, n_{i}-n_{i-1}}\right)$, where $\varepsilon_{i, j}=\varepsilon_{n_{i-1}+j}$ and $\sigma_{i}=\left(1 \ldots n_{i}-\right.$ $\left.n_{i-1}\right)$. Additionally, $\rho$ can be written as $\rho=\rho_{1} \oplus \ldots \oplus \rho_{t}$ where $\rho_{i}: \mathbb{Z}_{2}^{n-1} \rightarrow$ $\mathrm{GL}_{n_{i}-n_{i-1}}(\mathbb{Z})$ is a representation such that $\rho_{i}(x)$ is a diagonal matrix with \pm 1 on the diagonal. Then for all $x \in \mathbb{Z}_{2}^{n-1}$, we have that

$$
\begin{aligned}
\operatorname{det}(\mathbb{1}-\rho(x) D) & =\prod_{i=1}^{t} \operatorname{det}\left(\mathbb{1}-\rho_{i}(x) D_{i}\right) \\
& =\prod_{i=1}^{t} \operatorname{det}\left(\mathbb{1}-\rho_{i}(x) M_{\sigma_{i}}\left(\varepsilon_{i, 1}, \ldots, \varepsilon_{i, n_{i}-n_{i-1}}\right)\right) \\
& =\prod_{i=1}^{t}\left(1-\operatorname{det}\left(\rho_{i}(x)\right) \varepsilon_{i, 1} \ldots \varepsilon_{i, n_{i}-n_{i-1}}\right) .
\end{aligned}
$$

We now consider two cases. Suppose $M$ is not orientable. Then there exist $x^{-} \in \mathbb{Z}_{2}^{n-1}$ and $i \in\{1, \ldots, t\}$ such that $\operatorname{det}\left(\rho_{i}\left(x^{-}\right)\right)=-1$. If $\varepsilon_{i, 1} \ldots \varepsilon_{i, n_{i}-n_{i-1}}=$ 1 , we have that $\operatorname{det}(\mathbb{1}-\rho(0) D)=0$. If $\varepsilon_{i, 1} \ldots \varepsilon_{i, n_{i}-n_{i-1}}=-1$, we have that $\operatorname{det}\left(\mathbb{1}-\rho\left(x^{-}\right) D\right)=0$. We conclude that there exists $x \in \mathbb{Z}_{2}^{n-1}$ such that $\operatorname{det}(\mathbb{1}-$ $\rho(x) D)=0$. By Theorem 3.3, $R(f)=\infty$.

Suppose that $M$ is orientable, but that it is not homeomorphic to an HW manifold in standard form of which the associated graph is circulant. Then by Proposition 5.7 we have that $\sigma \neq(1 \ldots n)$, and hence $t>1$. Because $n$ is odd, there exists $i \in\{1, \ldots, t\}$ such that $n_{i}-n_{i-1}$ is odd. Pick $j \notin\left\{n_{i-1}+\right.$ $\left.1, \ldots, n_{i}\right\}$, we can do so because $t>1$. Pick $x^{-} \in \mathbb{Z}_{2}^{n-1}$ such that $\rho\left(x^{-}\right)=B_{j}$. Because the matrix $D_{i}=M_{\sigma_{i}}\left(\varepsilon_{i, 1}, \ldots, \varepsilon_{i, n_{i}-n_{i-1}}\right)$ has odd dimension it has a real eigenvalue. By Lemma 5.5, all eigenvalues of $D_{i}$ have modulus 1 , and hence $D_{i}$ has eigenvalue -1 or 1 . If $D_{i}$ has eigenvalue 1 , we have that $\operatorname{det}\left(\mathbb{1}-\rho_{i}(0) D_{i}\right)=0$, and hence $\operatorname{det}(\mathbb{1}-\rho(0) D)=0$. If $D_{i}$ has eigenvalue -1 , then $\rho_{i}\left(x^{-}\right) D_{i}=-D_{i}$ has eigenvalue 1 , and hence $\operatorname{det}\left(\mathbb{1}-\rho\left(x^{-}\right) D\right)=0$. So we always find $x \in \mathbb{Z}_{2}^{n-1}$ such that $\operatorname{det}(\mathbb{1}-\rho(x) D)=0$. By Theorem 3.3, $R(f)=\infty$.

Conversely suppose that $\Gamma$ is isomorphic to an HW group $\Gamma^{\prime}$ in standard form for which $\sigma=(1 \ldots n)$ is an automorphism of the graph associated to $\Gamma^{\prime}$. Without loss of generality, we may assume that $\Gamma$ is in standard form and that $\sigma$ is an automorphism of the graph associated to $\Gamma$. By Proposition 5.7 there exists $d \in \mathbb{R}^{n}$ such that $\left(d, M_{\sigma}(-1,1, \ldots, 1)\right) \in N_{\mathrm{Aff}\left(\mathbb{R}^{n}\right)}(\Gamma)$. Hence there exists $f: M \rightarrow M$ with homotopy lift $\left(d, M_{\sigma}(-1,1, \ldots, 1)\right)$. Now for all $x \in \mathbb{Z}_{2}^{n-1}$, we have that

$$
\operatorname{det}\left(\mathbb{1}-\rho(x) M_{\sigma}(-1,1, \ldots, 1)\right)=1-(-1) \cdot 1 \cdot \ldots \cdot 1=1-(-1)=2 \neq 0,
$$

and hence $R(f) \neq \infty$.

ExAmple 5.10. The classical Hantzsche-Wendt manifold does not have the $R_{\infty}$ property. Indeed, if we take the complement of its graph relative to 
the set $\{2\}$, we get a circulant graph:

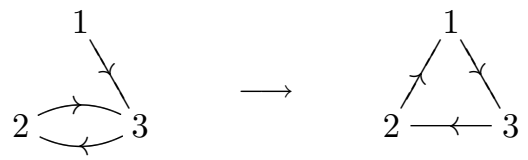

More generally, Miatello and Rossetti show in [16, Theorem 2.1] that for each odd dimension $n>1$, there exists an HW group of which the associated graph is the circulant graph $(V, E)$, where $V=\{1, \ldots, n\}$ and $(i, j) \in E$ if and only if $j-i \equiv 1 \bmod n$.

We conjecture that these are the only examples of HW manifolds that do not have the $R_{\infty}$ property.

Conjecture 5.11. In every odd dimension $n>1$, there is (up to homeomorphism) only one HW manifold that does not have the $R_{\infty}$ property. It is homeomorphic to the HW manifold in standard form of which the associated graph is $(V, E)$, where $V=\{1, \ldots, n\}$ and $(i, j) \in E$ if and only if $j-i \equiv$ $1 \bmod n$.

By a computer check, we can show this conjecture holds for all odd $n \leq 21$.

\section{REFERENCES}

[1] D. V. Anosov, The Nielsen numbers of maps of nil-manifolds, Uspekhi. Mat. Nauk 40 (1985), 133-134; English transl. Russian Math. Surveys 40 (1985), 149-150.

[2] K. S. Brown, Cohomology of Groups, Grad. Texts in Math., vol. 87, Springer-Verlag New York Inc., 1982

[3] R. F. Brown, The Lefschetz Fixed Point Theorem, Scott, Foresman and Company, 1971.

[4] K. Dekimpe, Almost-Bieberbach Groups: Affine and Polynomial Structures, Lecture Notes in Math., vol. 1639, Springer-Verlag, 1996.

[5] K. Dekimpe, B. De Rock And W. Malfait, The Anosov theorem for flat generalized Hantzsche-Wendt manifolds, J. Geom. Phys. 52 (2004), 174-185.

[6] The Nielsen numbers of Anosov diffeomorphisms on flat Riemannian manifolds, Forum Math. 17 (2005), 325-341.

[7] A. Fel'Shtyn and R. Hill, Dynamical zeta functions, Nielsen theory and Reidemeister torsion, Nielsen Theory and Dynamical Systems (South Hadley, MA, 1992), Contemp. Math., vol. 152, Amer. Math. Soc., 1993, pp. 43-68.

[8] A. L. Fel'Shtyn, R. Hill And P. Wong, Reidemeister numbers of equivariant maps, Topology Appl. 67 (1995), 119-131.

[9] D. L. Gonçalves and P. N.-S. Wong, Homogeneous spaces in coincidence theory II, Forum Math. 17 (2005), 297-313.

[10] B. Jiang, Nielsen Fixed Point Theory, Contemp. Math., vol. 14, Amer. Math. Soc., 1983.

[11] T. Kiang, The Theory of Fixed Point Classes, Springer-Verlag, 1989. 
[12] S. W. Kim, J. B. Lee And K. B. Lee, Averaging formula for Nielsen numbers, Nagoya Math. J. 178 (2005), 37-53.

[13] J. B. LEE AND K. B. LEE, Lefschetz numbers for continuous maps and periods for expanding maps on infra-nilmanifolds, J. Geom. Phys. 56 (2006), 2011-2023.

[14] K. B. LeE, Maps of infra-nilmanifolds, Pacific J. Math. 168 (1995), 157-166.

[15] A. Manning, There are no new Anosov diffeomorphisms on tori, Amer. J. Math. 96 (1974), 422-429.

[16] R. Miatello and J. P. Rossetti, Isospectral Hantzsche-Wendt manifolds, J. Reine Angew. Math. 515 (1999), 1-23.

[17] B. Norton-Odenthal, A product formula of generalized Lefschetz number, Ph. D. thesis (1991), University of Wisconsin-Madison.

[18] H. L. Porteous, Anosov diffeomorphisms of flat manifolds, Topology 11 (1972), 307315 .

[19] J. P. Rossetti And A. Szczepański, Generalized Hantzsche-Wendt flat manifolds, Rev. Mat. Iberoamericana 21 (2005), 1053-1070.

[20] J. TABACK AND P. Wong, A note on twisted conjugacy and generalized BaumslagSolitar groups, Preprint (2006).

Manuscript received December 21, 2007

KAREL DEKIMPE

Katholieke Universiteit Leuven

Campus Kortrijk

B-8500 Kortrijk, BELGIUM

E-mail address: Karel.Dekimpe@kuleuven-kortrijk.be

BRAM DE ROCK

Katholieke Universiteit Leuven

Campus Kortrijk

B-8500 Kortrijk, BELGIUM

Current address: Université Libre de Bruxelles

ECARES

B-1050 Brussels, BELGIUM

E-mail address: bDeRock@ulb.ac.be

Pieter Penninckx

Katholieke Universiteit Leuven

Campus Kortrijk

B-8500 Kortrijk, BELGIUM

E-mail address: Pieter.Penninckx@kuleuven-kortrijk.be 